\title{
The Use of Nonlinear Constitutive Equations to Evaluate Draw Resistance and Filter Ventilation*
}

\author{
by \\ B. Eitzinger ${ }^{1}$ and G. Ederer ${ }^{2}$ \\ ${ }^{1}$ WFT Research, Trierenberg Holding, Traun, Austria \\ ${ }^{2}$ Austria Tabak AG, Vienna, Austria
}

\section{SUMMARY}

This study investigates by nonlinear constitutive equations the influence of tipping paper, cigarette paper, filter, and tobacco rod on the degree of filter ventilation and draw resistance. Starting from the laws of conservation, the path to the theory of fluid dynamics in porous media and Darcy's law is reviewed and, as an extension to Darcy's law, two different nonlinear pressure drop-flow relations are proposed. It is proven that these relations are valid constitutive equations and the partial differential equations for the stationary flow in an unlit cigarette covering anisotropic, inhomogeneous and nonlinear behaviour are derived. From these equations a system of ordinary differential equations for the onedimensional flow in the cigarette is derived by averaging pressure and velocity over the cross section of the cigarette. By further integration, the concept of an electrical analog is reached and discussed in the light of nonlinear pressure drop-flow relations. By numerical calculations based on the system of ordinary differential equations, it is shown that the influence of nonlinearities cannot be neglected because variations in the degree of filter ventilation can reach up to $20 \%$ of its nominal value. [Beitr. Tabakforsch. Int. 19 (2000) 177-188)

\section{ZUSAMMENFASSUNG}

Diese Untersuchung beschäftigt sich mit dem Einfluß nichtlinearer Materialgesetze für Tipping, Zigarettenpapier, Filter und Tabakstrang auf den Filterventilationsgrad und den Zugwiderstand. Beginnend mit den Erhaltungsgleichungen wird der Weg zur Strömungsmechanik in porösen Medien und zu Darcy's Gesetz aufgezeigt. Als
Erweiterung von Darcy's Gesetz werden zwei nichtlineare Druck-Geschwindigkeits-Beziehungen vorgeschlagen. Es wird gezeigt, daß diese beiden Beziehungen tatsächlich Materialgesetze darstellen. Die partiellen Differentialgleichungen für die stationäre Strömung in nicht angezündeten Zigaretten unter Berücksichtigung von anisotropem, inhomogenem und nichtlinearem Verhalten werden abgeleitet. Ausgehend von diesen Gleichungen wird durch Durchschnittsbildung von Druck und Geschwindigkeit über den Querschnitt der Zigarette ein System gewöhnlicher Differentialgleichungen erzeugt, das die eindimensionale Strömung in der Zigarette beschreibt. Die weitere Integration der Gleichungen führt zum Konzept der elektrischen Analogie, das unter dem Gesichtspunkt nichtlinearer Verhältnisse diskutiert wird. Mittels numerischer Integration der gewöhnlichen Differentialgleichungen wird gezeigt, daß der Einfluß der Nichtlinearitäten nicht vernachlässigbar ist, da sich Variationen im Filterventilationsgrad von bis zu $20 \%$ des Nominalwerts ergeben. [Beitr. Tabakforsch. Int. 19 (2000) 177-188)

\section{RESUME}

Cette étude examine l'influence des principes non-linéaires propres aux matériaux du papier manchette, du papier à cigarettes, du filtre et du boudin de tabac sur le taux de ventilation $\mathrm{du}$ filtre et sur la résistance au tirage. A partir des équations de conservation, le processus de la mécanique des fluides dans des matériaux poreux et la loi de Darcy sont examinés. Comme extension de la loi de Darcy deux relations non-linéaires différentes entre perte de charge et débit d'air sont proposées. Il est montré que ces deux relations constituent des principes valides propres aux matériaux, et les équations différentielles par- 
tielles du courant stationnaire dans une cigarette non allumé, prenant en compte le comportement anisotrope, non-homogène et non-linéaire, en sont dérivées. En faisant - à partir de ces équations - la moyenne de la pression et de la vitesse par rapport à la section transversale de la cigarette, un système d'équations différentielles ordinaires est dérivé pour décrire l'écoulement d'air à une dimension dans la cigarette. L'intégration ultérieure de ces équations mène au concept de l'analogie électrique ce qui est discuté en tenant compte de relations perte de charge-débit d'air non-linéaires. L'intégration numérique des équations différentielles ordinaires démontre que l'influence des effets non-linéaires ne doit pas être négligée, parce que les variations du taux de ventilation du filtre peuvent atteindre $20 \%$ de la valeur nominale. [Beitr. Tabakforsch. Int. 19 (2000) 177-188)

\section{INTRODUCTION}

Since its early days in 1966 (4), until today (6), the theory concerning the stationary flow in unlit cigarettes has often dealt only with linear or linearized pressure drop-flow relations and thus is based on Darcy's law for the flow in porous media. Because of ever decreasing limits on specifications and the need to predict the degree of filter ventilation and draw resistances without costly experiments, the use of nonlinear constitutive equations could no longer be ignored and was thus investigated by several researchers (10-14). SELKE and MATHEWS in 1978 (5) and BAKER in 1989 (7) have shown on theoretical grounds and by experiments that the flow through tipping paper, cigarette paper, and the tobacco rod is intrinsically nonlinear due to inertial effects. It is the subject of this study to provide a theoretical foundation for the pressure drop-flow relations proposed in (5) and (7), to show that constitutive equations can be derived from these relations, and to investigate their influence on the stationary flow in unlit cigarettes.

\section{LAWS OF CONSERVATION}

To introduce the notations used in this paper and to be able to clearly state all the assumptions and simplifications along the way, this discussion starts with the four universal laws of conservation that are the foundation of the whole field of mechanics. There are four balance laws concerning mass,

$$
\frac{\partial \rho}{\partial t}+\nabla_{i}\left(\rho v^{i}\right)=0
$$

linear momentum,

$$
\rho \frac{\partial v^{i}}{\partial t}+\rho v^{j} \nabla_{j} v^{i}=\rho k^{i}+\nabla_{j} \sigma^{i j},
$$

angular momentum,

$$
\sigma^{i j}=\sigma^{j i},
$$

and energy.

$$
\rho \frac{\partial e}{\partial t}+\rho v^{i} \nabla_{i} e=\sigma^{i j} \nabla_{i} v^{j}-\nabla_{i} q^{i} .
$$

In order not to restrict the discussion to a particular coordinate system, the equations are stated as tensor equations, where $\rho$ denotes the density, $v^{i}$ the velocity, $k^{i}$ a body force, $\sigma^{i j}$ the stress tensor, $e$ the energy density, and $q^{i}$ the heat flux. A detailed introduction to tensor calculus may be found in (1). In these equations, and from now on, Einstein's summation convention is adopted. This convention states that when a suffix (superscript or subscript) appears twice in a term, summation with respect to this suffix is understood, and the range of summation is $1,2,3$. Thus, e.g.,

$$
v^{j} \nabla_{j} v^{i}=\sum_{j=1}^{3} v^{j} \nabla_{j} v^{i}
$$

A superscript denotes a contravariant index, while a subscript denotes a covariant index. The coordinates will be written as $x^{i}, i=1,2,3$. Additionally the covariant metric tensor $g_{i j}$ and the contravariant metric tensor $g^{i j}$ are defined by $d s^{2}=g_{i j} d x^{i} d x^{j}$ and $g_{k j} g^{k i}=\delta_{j}^{i}$, respectively, with $d s$ as the length of an infinitesimal line element, and $\delta_{j}^{i}=1$, if $i=j$, else 0 , as the Kronecker delta. The metric tensor is assumed to be positive definite. From the metric tensor the Christoffel symbols of first and second kind and finally an expression for the covariant derivative $\nabla_{i}$ of an invariant and a contravariant first order tensor can be derived. The Christoffel symbol of the first kind is defined as

$$
[i j, k]=\frac{1}{2}\left(\frac{\partial g_{k i}}{\partial x^{j}}+\frac{\partial g_{k j}}{\partial x^{i}}-\frac{\partial g_{i j}}{\partial x^{k}}\right) .
$$

The Christoffel symbol of the second kind can be obtained by raising an index

$$
\left\{\begin{array}{l}
k \\
i j
\end{array}\right\}=g^{k s}[i j, s] .
$$

It has to be noted that neither Christoffel symbol is a tensor. With these two symbols, a covariant derivative $\nabla_{i}$ can be defined such that the derivative of a tensor of arbitrary order will again give a set of quantities that transform according to the rules of tensor calculus.

$$
\begin{gathered}
T_{i}=\nabla_{i} p=\frac{\partial p}{\partial x^{i}} \\
T_{i}^{j}=\nabla_{i} v^{j}=\frac{\partial v^{j}}{\partial x^{i}}+\left\{\begin{array}{c}
j \\
k i
\end{array}\right\} v^{k} .
\end{gathered}
$$

Returning to the equations of conservation [1]-[4], it can be observed that the set of equations consists of only eight equations with a total of 17 unknowns. Thus additional equations and assumptions have to be introduced to obtain a unique solution. For the stationary flow in an 
unlit cigarette all time derivatives will vanish and, as the temperature is approximately constant, the energy balance decouples from the other equations and need not be considered further. The flow velocity is far below the speed of sound, so the flow can be treated as incompressible. At this point the equations are completely general and not related to any specific material, but, as there are still more variables than equations, a constitutive equation has to be added, relating the components of the stress tensor to the components of the strain tensor rate and thus to the velocity components. This constitutive equation is Newtonian fluid law (3),

$$
\sigma^{i j}=-p \delta^{i j}+\lambda \dot{\tau}^{k k} \delta^{i j}+2 \eta \dot{\tau}^{i j},
$$

where $p$ denotes the pressure. The rate of deformation tensor $\dot{\tau}^{i j}$ is defined by $\dot{\tau}^{i j}=1 / 2\left(g^{i k} \nabla_{k} v^{j}+g^{k j} \nabla_{k} v^{i}\right)$. For an incompressible fluid $\tau^{k k}=0$ and equation [9] simplifies to $\sigma^{i j}=-p \delta^{i j}+2 \eta \dot{\tau}^{i j}$. For our purpose air can be treated as a homogeneous ( $\rho=\eta=$ const.), Newtonian fluid leading to the Navier-Stokes equations for incompressible, stationary flow,

$$
\begin{aligned}
\rho v^{j} \nabla_{j} v^{i}-\eta g^{j k} \nabla_{j} \nabla_{k} v^{i} & =\rho k^{i}-g^{i j} \nabla_{j} p \\
\nabla_{i} v^{i} & =0 .
\end{aligned}
$$

The Navier-Stokes equations [10] and their solution for a straight pipe with circular cross section have often been used as a starting point to deal with flow problems in the cigarette $(8,9)$. However, calculating the velocity and pressure field inside a cigarette by the Navier-Stokes equations is a completely hopeless endeavour. In order to correctly state the problem, every single filter fiber, every piece of tobacco, and every perforation hole in the paper would have to be included in the boundary conditions.

Instead of dealing with the velocity around a single filter fiber, a continuum approach can be taken and only quantities averaged over a so called "representative elementary volume" are considered. The representative elementary volume has to be significantly larger than the thickness of a single fiber, but also small compared to the entire flow domain. This averaging process is described in detail by BEAR (2) and replaces the Navier-Stokes equation, by Darcy's law and the conservation of mass equation

$$
\begin{aligned}
v^{i} & =-k^{i j} \nabla_{j} p \\
\nabla_{i} v^{i} & =0 .
\end{aligned}
$$

The contravariant second-order tensor $k^{i j}$ is called tensor of hydraulic conductivity and represents the anisotropic behaviour. In the case of isotropy, the second order tensor degenerates to a scalar value. Similarly to other constitutive equations, e.g., the laws of Fourier, Ohm, Newton, and Hooke, Darcy's law provides a linear relation between the gradient of a driving force and the flux caused by the force. It is this linearity that has been thoroughly exploited, e.g., by translating the flow problem into a problem of electrical engineering or by giving simple closed form solutions of the differential equations. Nevertheless, several authors have stated that a linear law does not hold for perforated papers or the tobacco rod (5, 7), thus, if we wish to make precise predictions of draw resistances and degrees of filter ventilation, Darcy's law must be generalized to include nonlinear behaviour.

\section{TWO NONLINEAR VELOCITY-PRESSURE DROP RELATIONS}

SELKE and MATHEWS (5) and BAKER (7) proposed two different velocity-pressure drop relations, which both gave excellent agreement with experimental data

$$
\begin{gathered}
v(\Delta p)=k \Delta p^{n} \\
v(\Delta p)=k_{1} \Delta p+k_{2} \sqrt{\Delta p}
\end{gathered}
$$

An obvious generalization of equations [12] and [13] to three dimensions might be

$$
\begin{gathered}
v^{i}\left(\nabla_{j} p\right)=k\left(g^{i j} \nabla_{j} p\right)^{n} \\
v^{i}\left(\nabla_{j} p\right)=k_{1} g^{i j} \nabla_{j} p+k_{2} \sqrt{g^{i j} \nabla_{j} p}
\end{gathered}
$$

Unfortunately not every function $v^{i}\left(\nabla_{j} p\right): \mathbb{R}^{3} \rightarrow \mathbb{R}^{3}$ is a constitutive equation. First, the tensorial properties of $v^{i}$ have to be conserved under general coordinate transformations. Let us assume that $a_{i}$ is a vector (first order tensor) and we want to investigate the properties of $b_{i}=$ $\left(a_{i}\right)^{n}$. If $b_{i}$ were also a vector, then by passing from a system of coordinates $x^{i}$ to $x^{/ i}$ we should have $b_{i}=\left(a_{i}\right)^{n}$ and $b_{i}^{\prime}=\left(a_{i}^{\prime}\right)^{n}$. But in the nontrivial case $n \neq 1$, we have $b_{i}^{\prime}=\frac{\partial x^{j}}{\partial x^{/ i}} b_{j}=\frac{\partial x^{j}}{\partial x^{/ i}}\left(a_{j}\right)^{n} \neq\left(\frac{\partial x^{j}}{\partial x^{/ i}} a_{j}\right)^{n}=\left(a_{i}^{\prime}\right)^{n}$ and therefore, $b_{i}=\left(a_{i}\right)^{n}$ is not a vector. Consequently equations [14] and [15] are not constitutive equations. In addition, it is necessary in order not to violate the second law of thermodynamics, that $v^{i} \nabla_{i} p \leq 0$. This is equal to demanding that the pressure gradient and the velocity always have to form an obtuse angle. If this condition is not satisfied, air would be allowed to flow by itself into regions of higher pressure and, although this is consistent with the energy balance, it violates the second law of thermodynamics. Equations [14] and [15] do not satisfy this condition, because in the general case $\left(\nabla_{j} p\right)^{n} \notin \mathbb{R}$ for $\nabla_{j} p<0$. Secondly, it is desirable, though not necessary, that even in the anisotropic case for every $b \in \mathbb{R}$, there should exist an $b^{\prime} \in \mathbb{R}$ such that $v^{i}\left(b \nabla_{j} p\right)=b^{\prime} v^{i}\left(\nabla_{j} p\right)$. This condition states that raising the pressure gradient from $\nabla_{i} p$ to $b \nabla_{i} p$ only changes the length of the velocity vector and not its direction, because the anisotropy, and thus the angle between pressure gradient and velocity, is a geometric property of the solid, approximately rigid 
body and should not depend on the pressure gradient. It can easily be proven that this condition does not hold for equation [15].

\section{A NEW CONSTITUTIVE EQUATION}

To begin, a new nonlinear constitutive equation is proposed and, as stated by BEAR (2) and SPURK (3), six conditions are given, which must be satisfied by all constitutive equations. Compliance with these conditions will then be investigated.

As an extension of Darcy's law a new constitutive equation is proposed by including an invariant function $f\left(\nabla_{j} p\right): \mathbb{R}^{3} \rightarrow \mathbb{R}^{+}$in the constitutive equation

$$
v^{i}=-k^{i j} f\left(\nabla_{j} p\right) \nabla_{j} p
$$

with $k^{i j}$ and $f\left(\nabla_{j} p\right)$ positive semidefinite.

\section{Condition 1: Consistency}

The constitutive equation [16] has to be consistent with the balance laws [1]-[4] and the second law of thermodynamics (entropy), but it must not be a consequence of these laws. Together with the laws of conservation, the constitutive equation has to provide a system of equations, for which the existence and uniqueness of a solution can be guaranteed. It can be tested by Frobenius' theorem, whether or not a unique solution exists for a given system of partial differential equations. Because of the length of the necessary calculations, this matter is not investigated herein. To comply with the second law of thermodynamics, it is necessary and sufficient that $v^{i} \nabla_{i} p \leq 0$. Thus $-f\left(\nabla_{i} p\right) k^{i j} \nabla_{j} p \nabla_{i} p \leq 0$, which is satisfied as $k^{i j}$ and $f\left(\nabla_{i} p\right)$ are positive semidefinite. Additionally $k^{i j}$ is symmetric due to its positive semidefiniteness, and also due to physical reasons (2).

\section{Condition 2: Independence}

It must be possible to state the law independently of any particular coordinate system. This is usually done by stating the equation as a tensor equation, and it is the main reason for the use of tensor calculus in this study. Equation [16] is a tensor equation and thus satisfies condition 2.

\section{Condition 3: Determinism}

The history of the pressure up to time $t$ uniquely determines the velocity at time $t$ and thus equation [16] is deterministic.

\section{Condition 4: Local validity}

The velocity at point $x^{i}$ has to depend only on the pressure in the immediate neighborhood of $x^{i}$. Equation
[16] fulfils this condition, while, e.g., equation [12] does not.

\section{Condition 5: Symmetry}

The symmetry properties of the material have to be contained in the constitutive equation. Thus, if the material is invariant with respect to certain coordinate transformations, the constitutive equation must also be invariant with respect to these transformations. In equation [16], the anisotropy is contained in the second order tensor $k^{i j}$ and, as the function $f\left(\nabla_{i} p\right)$ is an invariant and not tensor-valued, the symmetry properties will only depend on $k^{i j}$, just as they do in Darcy's law.

\section{Condition 6: Frame independence}

As is well known, the conservation equations [1]-[4] are not frame independent. In accelerating reference frames, the apparent forces, e.g., centrifugal and Coriolis forces, are introduced, and condition 6 ensures that this remains the only difference for the transition from an inertial system to a relative system (3). Equation [16] does not satisfy this condition because the velocity is not a frame independent ("objective") quantity. This problem can be overcome by introducing a co-rotational time derivative (15). Strictly speaking, equation [16] is not a valid constitutive equation. But for the description of the flow in cigarettes, accelerating reference frames are hardly ever used. Thus, we avoid the complications of this time derivative, and accept the relation as valid for all practical purposes.

As equation [16] has been proven to be a constitutive equation, the function $f\left(\nabla_{i} p\right)$ still remains to be specified. Let us assume that $f\left(\nabla_{i} p\right)$ depends only on the squared length of $\nabla_{i} p$, thus $f\left(\nabla_{i} p\right)=f\left(g^{i j} \nabla_{i} p \nabla_{j} p\right)$. To obtain a final version of the nonlinear constitutive equation, the function $f\left(\nabla_{i} p\right)$ is chosen so as to reflect the structure of equations [12] and [13].

$$
\begin{gathered}
v^{i}=-k^{i j}\left(g^{q s} \nabla_{q} p \nabla_{s} p\right)^{\frac{n-1}{2}} \nabla_{j} p \\
v^{i}=-k^{i j}\left[1+b \cdot\left(g^{q s} \nabla_{q} p \nabla_{s} p\right)^{-\frac{1}{4}}\right] \nabla_{j} p
\end{gathered}
$$

A big advantage of these constitutive equations is that they contain only one additional parameter $n$ and $h$, respectively, which have to be determined by measurements. Together with the conservation of mass equation [1], a single partial differential equation can be derived, which describes incompressible flow in inhomogeneous porous media. For the two constitutive equations [17] and [18], these equations are

$$
\nabla_{i}\left[k^{i j}\left(g^{q s} \nabla_{q} p \nabla_{s} p\right)^{\frac{n-1}{2}} \nabla_{j} p\right]=0
$$

and 


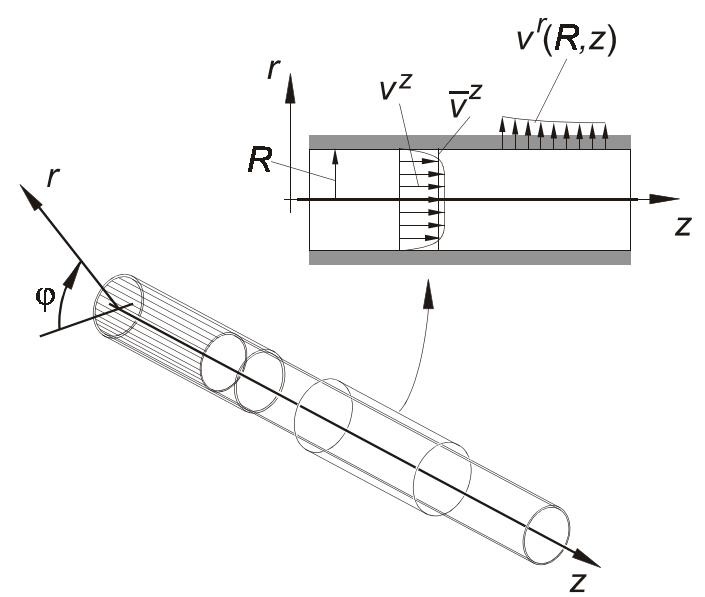

Figure 1.

The cylindrical polar coordinate system for the cigarette. The detail shows the velocity profile $v^{z}$ in $z$-direction, the average velocity $\bar{v}^{z}$, and the radial velocity $v^{r}(R, z)$ at the outer boundary.

$$
\nabla_{i}\left[k^{i j}\left[1+b\left(g^{q s} \nabla_{q} p \nabla_{s} p\right)^{-\frac{1}{4}}\right] \nabla_{j} p\right]=0
$$

It has to be noted that equations [19] and [20] include the papers as well as the filter and the tobacco rod. Usually, pressure drop-flow relations for the paper are given as $v(\Delta p)$ and for the filter as $\Delta p(v)$, but as far as the partial differential equations are concerned, this distinction is completely artificial. It only obscures the fact that both relations represent the same phenomenon, i.e., the flow in porous media. Later on, for the ordinary differential equations, this distinction is again useful, but only for computational and not for physical reasons.

In general, solutions to equation [19] or [20] can only be found by numerical methods, but the problem arising from the nonlinearity of the differential equation is beyond the scope of this investigation. The first simplification of equations [19] and [20] can be found by averaging over the cross section of the cigarette. This results in a system of ordinary differential equations, which finally, though not trivially, can be solved. From now on in this discussion, only constitutive equation [17] will be considered. Of course the calculations can be repeated for the second constitutive equation, and will yield similar results.

\section{THE ONE-DIMENSIONAL FLOW PROBLEM}

So far the discussion has not been restricted to a specific coordinate system. But in order to carry out the averaging process, the calculations can be considerably simplified by choosing a suitable coordinate system. Cylindrical polar coordinates are the obvious choice for the cigarette. Figure 1 shows the coordinate system.

The length of the line element in cylindrical polar coordinates is $d s^{2}=d r^{2}+r^{2} d \varphi^{2}+d z^{2}$ and the metric tensors are

$$
\begin{aligned}
& g_{r r}=g_{z z}=1, g_{\varphi \varphi}=r^{2}, g_{i j}=0 \text { if } i \neq j \\
& g^{r r}=g^{z z}=1, g^{\varphi \varphi}=r^{-2}, g^{i j}=0 \text { if } i \neq j .
\end{aligned}
$$

For the tensor of hydraulic conductivity $k^{i j}$, there exists a system of three orthogonal principal axes, in which all non-diagonal elements of $k^{i j}$ vanish. It is reasonable to assume, that one of these axes is aligned with the rotational axis of the cigarette, and thus the $z$-axis of the coordinate system. The coordinate system is then chosen such that the other two axes coincide with the principal axes. The tensor of hydraulic conductivity $k^{i j}$ can then be written in a diagonal form, i.e., $k^{i j}=0$ if $i \neq j$. For our coordinate system, the Christoffel symbols of first kind evaluate to $[r \varphi, \varphi]=[\varphi r, \varphi]=r$ and $[\varphi \varphi, r]=-r$ by equation [5]; all other components vanish. The Christoffel symbols of the second kind, calculated by equation [6], are

$$
\left\{\begin{array}{c}
\varphi \\
r \varphi
\end{array}\right\}=\left\{\begin{array}{c}
\varphi \\
\varphi r
\end{array}\right\}=\frac{1}{r},\left\{\begin{array}{c}
r \\
\varphi \varphi
\end{array}\right\}=-r .
$$

Again, all the other components vanish. With equation [8] for the covariant derivative of a first order tensor, we obtain the conservation of mass equation in contravariant velocity components for cylindrical polar coordinates.

$$
\begin{aligned}
\nabla_{i} \boldsymbol{v}^{i} & =\frac{\partial v^{i}}{\partial x^{i}}+\left\{\begin{array}{c}
i \\
k i
\end{array}\right\} v^{k} \\
& =\frac{\partial v^{r}}{\partial r}+\frac{\partial v^{\varphi}}{\partial \varphi}+\left\{\begin{array}{c}
\varphi \\
r \varphi
\end{array}\right\} v^{r}+\frac{\partial v^{z}}{\partial z} \\
& =\frac{\partial v^{r}}{\partial r}+\frac{1}{r} v^{r}+\frac{\partial v^{\varphi}}{\partial \varphi}+\frac{\partial v^{z}}{\partial z} \\
& =\frac{1}{r} \frac{\partial}{\partial r}\left(r v^{r}\right)+\frac{\partial v^{\varphi}}{\partial \varphi}+\frac{\partial v^{z}}{\partial z}=0
\end{aligned}
$$

By applying equation [7] to constitutive equation [17], we obtain in contravariant components

$$
\begin{aligned}
& v^{r}=-k^{r r}\left(p_{r}^{2}+r^{-2} p_{\varphi}^{2}+p_{z}^{2}\right)^{\frac{n-1}{2}} p_{r} \\
& v^{\varphi}=-k^{\varphi \varphi}\left(p_{r}^{2}+r^{-2} p_{\varphi}^{2}+p_{z}^{2}\right)^{\frac{n-1}{2}} p_{\varphi} \\
& v^{z}=-k^{z z}\left(p_{r}^{2}+r^{-2} p_{\varphi}^{2}+p_{z}^{2}\right)^{\frac{n-1}{2}} p_{z}
\end{aligned}
$$

with

$$
p_{r}=\frac{\partial p}{\partial r}, p_{\varphi}=\frac{\partial p}{\partial \varphi}, p_{z}=\frac{\partial p}{\partial z} .
$$

While contravariant components are a mathematically convenient way to express these relations, they have in general no direct physical meaning. Indeed they may have physical dimensions different from those of the physical quantity they express (1). Noting that the contravariant and covariant unit vectors are

and

$$
\mathbf{e}^{r}=\left[\begin{array}{l}
1 \\
0 \\
0
\end{array}\right], \mathbf{e}^{\varphi}=\left[\begin{array}{c}
0 \\
r^{-1} \\
0
\end{array}\right], \mathbf{e}^{z}=\left[\begin{array}{l}
0 \\
0 \\
1
\end{array}\right]
$$




$$
\mathbf{e}_{r}=\left[\begin{array}{l}
1 \\
0 \\
0
\end{array}\right], \mathbf{e}_{\varphi}=\left[\begin{array}{l}
0 \\
r \\
0
\end{array}\right], \mathbf{e}_{z}=\left[\begin{array}{l}
0 \\
0 \\
1
\end{array}\right],
$$

respectively, we could convert equations [21] and [22] to physical (normalized) components by

$$
\begin{gathered}
\mathbf{v}=v^{i} \mathbf{e}_{i}=\left[\begin{array}{c}
v^{r} \\
r v^{\varphi} \\
v^{z}
\end{array}\right], \\
\operatorname{grad} p=\nabla_{i} p \mathbf{e}^{i}=\left[\begin{array}{c}
p_{r} \\
r^{-1} p_{\varphi} \\
p_{z}
\end{array}\right],
\end{gathered}
$$

and

$$
\mathbf{k}=k^{i j} \mathbf{e}_{i} \mathbf{e}_{j}=\left[\begin{array}{ccc}
k^{r r} & 0 & 0 \\
0 & r^{2} k^{\varphi \varphi} & 0 \\
0 & 0 & k^{z z}
\end{array}\right] .
$$

A major simplification can be achieved by assuming rotational symmetry, i.e., $p_{\varphi}=\partial p / \partial \varphi=0$, and thus $v^{\varphi}=0$. As can be seen in equations [23]-[25], the only difference between the contravariant and the physical description is in the $\varphi$-component, so in a rotationally symmetric flow domain there is no need to distinguish between these two descriptions.

Next, an average quantity (pressure or velocity) $\bar{u}(z)$ is defined by averaging its value $u(r, z)$ over the cross section of the cigarette

$$
\bar{u}(z)=\frac{1}{\pi R^{2}} \int_{0}^{R} u(r, z) \cdot 2 \pi r d r,
$$

where $R$ denotes the radius of the cigarette and the overbar signifies the averaging process. It is not self-evident that the averaging process and the application of a function can be interchanged, i.e., $\overline{f(u(r, z))}=f(\bar{u}(z))$, thus the averaging process has to be looked at in detail

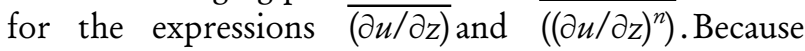
differentiation and integration are linear operations and can be interchanged, it can easily be proven that

$$
\overline{\left(\frac{\partial u}{\partial z}\right)}=\frac{1}{\pi R^{2}} \int_{0}^{R} \frac{\partial u}{\partial z}(r, z) \cdot 2 \pi r d r=\frac{\partial \bar{u}}{\partial z} .
$$

On the other hand, it is clear that $\overline{\left((\partial u / \partial z)^{n}\right)} \neq(\partial \bar{u} / \partial z)^{n}$ even with further assumptions. To ensure that at least $\left((\partial u / \partial z)^{n}\right)=\alpha \cdot(\partial \bar{u} / \partial z)^{n}, \alpha \in \mathbb{R}$, it is necessary to assume that the quantity $u(r, z)$ can be decomposed into the product of two functions $u(r, z)=f_{1}(z) \cdot f_{2}(r)$. Then it can be found that

$$
\frac{\partial \bar{u}}{\partial z}=\frac{1}{\pi R^{2}} \int_{0}^{R} \frac{\partial f_{1}(z)}{\partial z} f_{2}(r) \cdot 2 \pi r d r=\alpha_{1} \frac{\partial f_{1}(z)}{\partial z}
$$

and

$$
\begin{aligned}
\overline{\left(\frac{\partial u}{\partial z}\right)^{n}} & =\frac{1}{\pi R_{2}} \int_{0}^{R}\left(\frac{\partial f_{1}(z)}{\partial z}\right)^{n} f_{2}^{n}(r) \cdot 2 \pi r d r \\
& =\alpha_{n}\left(\frac{\partial f_{1}(z)}{\partial z}\right)^{n}=\frac{\alpha_{n}}{\alpha_{1}^{n}}\left(\frac{\partial \bar{u}}{\partial z}\right)^{n}=\alpha\left(\frac{\partial \bar{u}}{\partial z}\right)^{n} .
\end{aligned}
$$

It might be objected that the function $f_{2}(r)$ is a priori unknown, and that $\alpha_{1}$ and $\alpha_{n}$ cannot be calculated. It will be shown, however, that the coefficient $\alpha$ can be included in the material parameters that are determined by measurements, and will not appear in the equations explicitly. Applying the averaging procedure to the conservation of mass [21], the integration gives

$$
\frac{1}{\pi R^{2}} \int_{0}^{R} r^{-1} \frac{\partial}{\partial r}\left(r v^{r}\right) \cdot 2 \pi r d r+\frac{1}{\pi R^{2}} \int_{0}^{R} \frac{\partial}{\partial z}\left(v^{z}\right) \cdot 2 \pi r d r=0
$$

$$
\begin{gathered}
\left.\frac{2}{R^{2}} r v^{r}\right|_{0} ^{R}+\frac{\partial \bar{v}^{z}}{\partial z}=0 \\
\frac{2}{R} v^{r}(R, z)+\frac{\partial \bar{v}^{z}(z)}{\partial z}=0
\end{gathered}
$$

This procedure can also be carried out for the constitutive equation [22], but as can be seen in equation [27], only the radial velocity $v^{r}(R, z)$ at the outer boundary of the cigarette and the average velocity in $z$-direction are of interest. By assuming $v^{z}(R, z)=v^{\varphi}(R, z)=0$, which is a reasonable assumption because the air will be flowing in an almost radial direction through the paper,

$$
v^{r}(R, z)=-k^{r r}\left|p_{r}\right|^{n-1} p_{r} .
$$

For reasons of mass conservation, the flow of air from the paper into the tobacco rod equals the flow of air through the paper, and thus equation [28] can be reformulated with the hydraulic conductivity of the paper, given by $\tilde{k}_{p}^{r r}$ and $n_{P}$, and the pressure gradient inside the paper denoted by $p_{r}{ }^{P}$.

$$
\begin{aligned}
v^{r}(R, z) & =-k^{r r}\left|p_{r}\right|^{n-1} p_{r}=-\tilde{k}_{P}^{r r}\left|p_{r}^{P}\right|^{n_{P}-1} p_{r}^{P} \\
& \approx-\frac{\tilde{k}_{P}^{r r}}{d^{n_{P}}}\left|p_{\infty}-\bar{p}(z)\right|^{n_{P}-1}\left(p_{\infty}-\bar{p}(z)\right) \\
& =-k_{P}^{r r}\left|p_{\infty}-\bar{p}(z)\right|^{n_{P}-1}\left(p_{\infty}-\bar{p}(z)\right),
\end{aligned}
$$

where $p_{\infty}$ is the environmental air pressure and $d$ is the thickness of the paper, which need not be known, because the factor $k_{P}^{r r}$ can be determined by the standard method of measuring air permeability. By considering the assumption [26], i.e., $p_{z}(z, r)=f_{1}(z) f_{2}(r)$, one gets for the average velocity $\bar{v}^{z}$

$$
\begin{aligned}
\bar{v}^{z} & =-\frac{k^{z z}}{\pi R^{2}} \int_{0}^{R}\left|\frac{\partial p}{\partial z}\right|^{n-1}\left(\frac{\partial p}{\partial z}\right) 2 \pi r d r \\
& =-k^{z z} \alpha\left|\bar{p}_{z}\right|^{n-1} \bar{p}_{z} .
\end{aligned}
$$




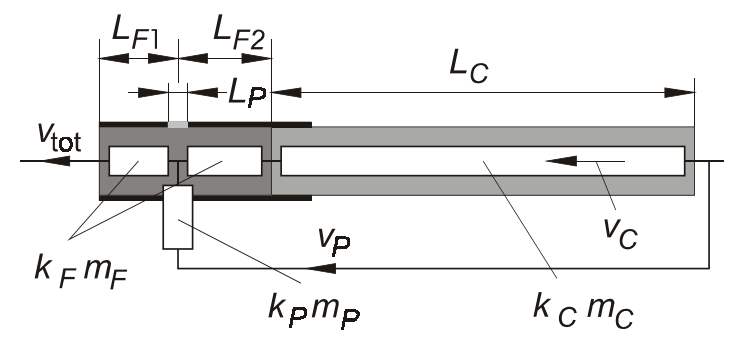

Figure 2.

The electrical analog of a cigarette; $k$ and $m$ denote parameters of the constitutive equations

The assumptions made here are $p_{r}^{2} \ll p_{z}^{2}, k^{z z}=$ const., and that $p_{z}$ does not change its sign over the range of integration. All simplifications are reasonable, because the flow inside the cigarette will be almost uniform and parallel to the $z$-axis over the whole cross section.

It is common practice instead of dealing with absolute pressures to only consider pressure differences, i.e., $p(z)=p_{\infty}-\bar{p}(z)$. Combining the conservation of mass equation [27] with the constitutive equation, renaming all the constants, leaving out the overbar, and substituting $p$ for the absolute pressure $\bar{p}$ leads to an implicit system of two ordinary, nonlinear differential equations.

$$
\begin{aligned}
\frac{\partial v^{z}}{\partial z} & =\frac{2}{R} k_{P}^{r r}|p|^{n_{P}-1} p \\
v^{z} & =k^{z z}\left|\frac{\partial p}{\partial z}\right|^{n-1} \frac{\partial p}{\partial z} .
\end{aligned}
$$

While a numerical treatment of system [31] is indeed possible, the equations are much more amenable to analytical computations, if they are given in explicit form. Such a form can be obtained by direct inversion of the second equation, renaming $k^{z z}$ and substituting $m=$ $n^{-1}$. It is also practical to substitute $-v^{z}$ for $v^{z}$ because in most cases the air will be flowing into the negative $z$ direction. This leads to a final version of the differential equations for constitutive equation [22].

$$
\begin{aligned}
\frac{\partial v^{z}}{\partial z} & =-\frac{2}{R} k_{P}^{r r}|p|^{n_{P}-1} p \\
\frac{\partial p}{\partial z} & =-k^{z z}\left|v^{z}\right|^{m-1} v^{z} .
\end{aligned}
$$

\section{A SOLUTION TO THE DISCRETIZED FLOW PROBLEM}

Figure 2 shows the electrical analog of a cigarette, where the filter, the perforation zone, and the tobacco rod are interpreted as resistances.

If the flow velocity is constant, the second differential equation of system [32] can be integrated for a pipe of length $L$.

$$
\begin{aligned}
\int_{0}^{L} \frac{\partial p}{\partial z} d z & =\int_{0}^{L}-k^{z z}\left|v^{z}\right|^{m-1} v^{z} d z \\
p(L)-p(0) & =-k^{z z} L\left|v^{z}\right|^{m-1} v^{z} .
\end{aligned}
$$

This expression is the nonlinear correspondence to Ohm's law, where $R=k^{z z} L$ and $m=1$. Considering Kirchhoff's rules, which are independent of the constitutive equation, an expression can be derived for the degree of filter ventilation. The pressure drop across each resistance for the pressure drop-flow relation [33] is given by

$$
\begin{aligned}
\Delta p_{F 1} & =k_{F} v_{\text {tot }}^{m_{F}} L_{F 1} \\
\Delta p_{F 2} & =k_{F} v_{C}^{m_{F}} L_{F 2} \\
\Delta p_{C} & =k_{C} v_{C}^{m_{C}} L_{C} \\
\Delta p_{P} & =k_{P} v_{P}^{m_{P}} .
\end{aligned}
$$

In the last equation, the thickness of the tipping paper has been included in the resistance $k_{P}$ because this resistance can easily be calculated from the standard method of measuring air permeability. Kirchhoff's rules give

$$
\begin{aligned}
\pi R^{2} v_{\text {tot }} & =\pi R^{2} v_{C}+2 \pi R L_{P} v_{P} \\
\Delta p_{P} & =\Delta p_{C}+\Delta p_{F 2} .
\end{aligned}
$$

With the definition of the degree of filter ventilation as $V_{F}=2 \pi R L_{P} v_{P} / \pi R^{2} v_{\text {tot }}$ one gets

$$
\begin{aligned}
\left(\frac{R}{2 L_{P}}\right)^{m_{P}} k_{P} v_{\text {tot }}^{m_{P}} V_{F}^{m_{p}}= & k_{F} v_{\text {tot }}^{m_{F}}\left(1-V_{F}\right)^{m_{F}} L_{F 2} \\
& +k_{C} v_{\text {tot }}^{m_{C}}\left(1-V_{F}\right)^{m_{C}} L_{C} .
\end{aligned}
$$

Clearly, this equation cannot be solved for $V_{F}$ in closed form. Additionally, the degree of filter ventilation depends on the flow velocity because in the general case $v_{\text {tot }}$ cannot be cancelled out. Assuming that $V_{F}$ is known, the draw resistance can be calculated simply by

$$
\begin{aligned}
\Delta p_{\text {tot }}= & k_{F} v_{\text {tot }}^{m_{F}} L_{F 1}+k_{F} v_{\text {tot }}^{m_{F}}\left(1-V_{F}\right)^{m_{F}} L_{F 2} \\
& +k_{C} v_{\text {tot }}^{m_{C}}\left(1-V_{F}\right)^{m_{C}} L_{C} .
\end{aligned}
$$

Again a deviation from linear theory can be observed, because the draw resistance [35] is not proportional to the flow velocity.

In equation [34], numerical methods are necessary to calculate the degree of filter ventilation. If there is no other way than to use the whole methodology of numerical mathematics, it seems more reasonable to calculate a solution of the differential equations [32], instead of calculating with great precision the solution of equation [34], which by itself is only an approximation of the original problem. 

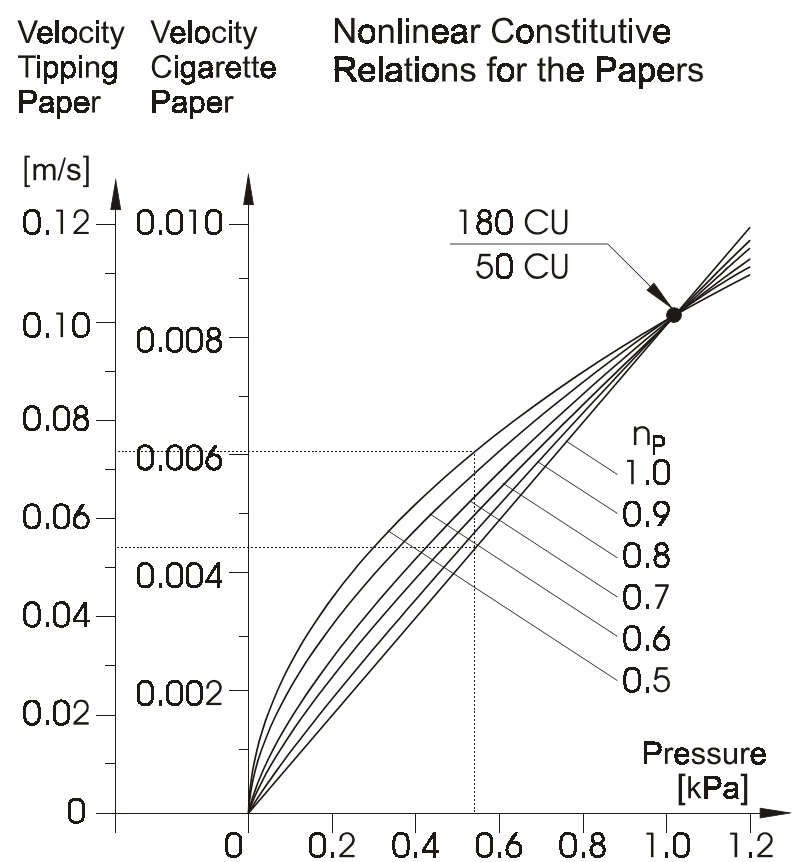

Figure 3.

Constitutive equations for the papers on the cigarette for various exponents $n_{p}$. The dotted lines show the velocity variation due to the nonlinearity for the actual pressure at the perforation zone.

\section{RESULTS}

As the effect of nonlinearities in the constitutive equations cannot be isolated for a real cigarette, the influence was investigated solely by numerical calculations. The fictitious cigarette chosen for the calculations had the following design parameters

Filter length:

$23 \mathrm{~mm}$

Filter pressure drop:

$70 \mathrm{~mm} \mathrm{H}_{2} \mathrm{O}$

Tobacco rod length:

$61 \mathrm{~mm}$

Tobacco rod pressure drop:

$60 \mathrm{~mm} \mathrm{H}_{2} \mathrm{O}$

Cigarette paper permeability:

Tipping paper length:

50 CORESTA units (CU)

$27 \mathrm{~mm}$

Tipping paper permeability:

Position of the perforation:

Cigarette diameter:

$180 \mathrm{CU}$

$12-15 \mathrm{~mm}$

$7.9 \mathrm{~mm}$

$84 \mathrm{~mm}$

The plug wrap paper was assumed to be of a sufficiently high permeability so that its influence on the flow through the tipping paper is negligible. It must be noted here, that for the calculations all these parameters were kept constant and the only point modified was the constitutive equation of one of the cigarette components. The cigarette described by these parameters will have a degree of filter ventilation of about $25 \%$ and a draw resistance of about $102.5 \mathrm{~mm} \mathrm{H}_{2} \mathrm{O}$.

In order to integrate the system of equations [32], a second order Runge-Kutta method was chosen, whose consistency and stability can be proven. Convergence

$\begin{array}{lll}\text { Pressure } & \text { Pressure } & \text { Nonlinear Constitutive } \\ \text { Drop } & \text { Drop } & \text { Relations for Filter and Rod } \\ \text { Filter } & \text { Rod } & \end{array}$

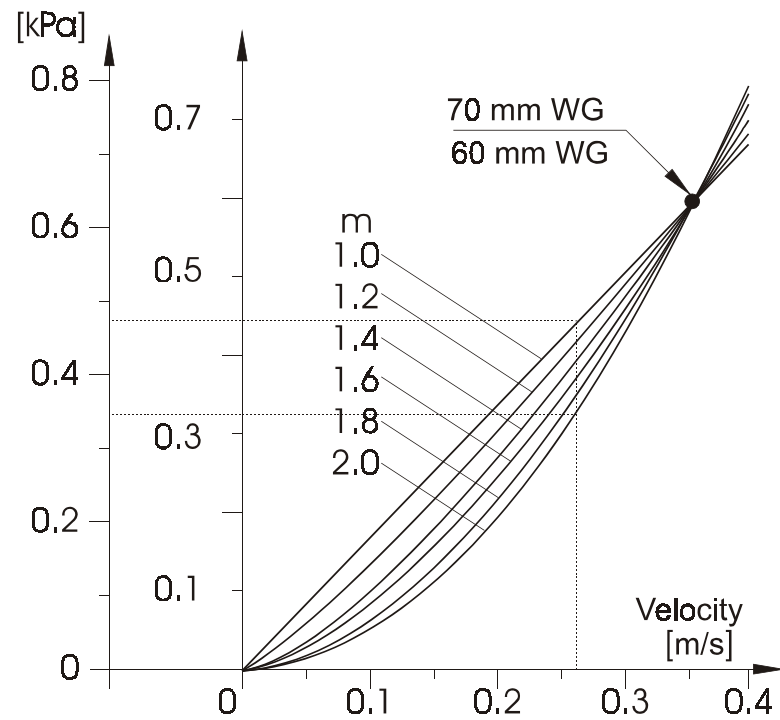

Figure 4.

Constitutive equations for the filter and the tobacco rod for various exponents $m$. The dotted lines show the pressure variation due to the nonlinearity for the flow velocity upstream of the perforation zone, i.e. reduced by the filter ventilation.

was verified by varying the number of discretization points. The problem to be solved is not an initial condition problem, but a boundary value problem, because on the mouth end of the cigarette (at $z=0$ ), the volumetric flow is given (assumed to be $v_{\text {tot }}=17.5 \mathrm{~cm}^{3} / \mathrm{s}$ ) while on the other end of the cigarette (at $z=84$ ), the pressure is fixed at environmental air pressure. To solve this boundary value problem, an iterative process was started by using a reasonable guess $p_{\text {mouth }}$ for the pressure at the mouth end as an initial condition, integrating the differential equations along the cigarette from $z=0 \mathrm{~mm}$ to $z=$ $84 \mathrm{~mm}$, and then updating the pressure $p_{\text {mouth }}$ according to the difference between the resulting pressure $p(z=84)$ and the environmental air pressure $p_{\infty}$ by $p_{\text {mouth,new }}=$ $p_{\text {mouth }}+p_{\infty}-p(z=84)$. The difference $p_{\infty}-p(z=84)$ can be reduced below $1 \mathrm{~Pa}(\approx 1 \%$ of the draw resistance) within a few iterations.

The constitutive equations used for the papers are shown in Figure 3. The coefficient $n_{P}$ was taken from the range 1.0 down to 0.5 , because this is the range that gives good agreement with real papers (5). All the tipping and cigarette papers would have been found to have a permeability of $180 \mathrm{CU}$ and $50 \mathrm{CU}$, respectively, because this quantity is measured at a pressure difference of $1 \mathrm{kPa}$. An important point needs to be stressed here if the perforation of the tipping paper is to be treated as a distributed property. The standard method of measuring air permeability uses a $20 \times 10 \mathrm{~mm}^{2}$ measurement area, but for a given perforation width $L_{p}$, the air permeable area is only $20 \times L_{p} \mathrm{~mm}^{2}$, and thus the actual flow velocity at the 
Nonlinear Constitutive Relation Tipping Paper

\begin{tabular}{|c|c|}
\hline$n_{P}$ & $\begin{array}{c}\text { Degree of } \\
\text { Filter Ventilation }\end{array}$ \\
\hline 1.0 & $25.7 \%$ \\
0.9 & $26.8 \%$ \\
0.8 & $27.8 \%$ \\
0.7 & $29.0 \%$ \\
0.6 & $30.3 \%$ \\
0.5 & $31.8 \%$ \\
\hline & Draw \\
$n_{P}$ & Resistance \\
\hline 1.0 & $102.5 \mathrm{~mm}$ WG \\
0.9 & $101.7 \mathrm{~mm}$ WG \\
0.8 & $100.8 \mathrm{~mm}$ WG \\
0.7 & $99.9 \mathrm{~mm}$ WG \\
0.6 & $98.8 \mathrm{~mm}$ WG \\
0.5 & $97.6 \mathrm{~mm}$ WG \\
\hline
\end{tabular}

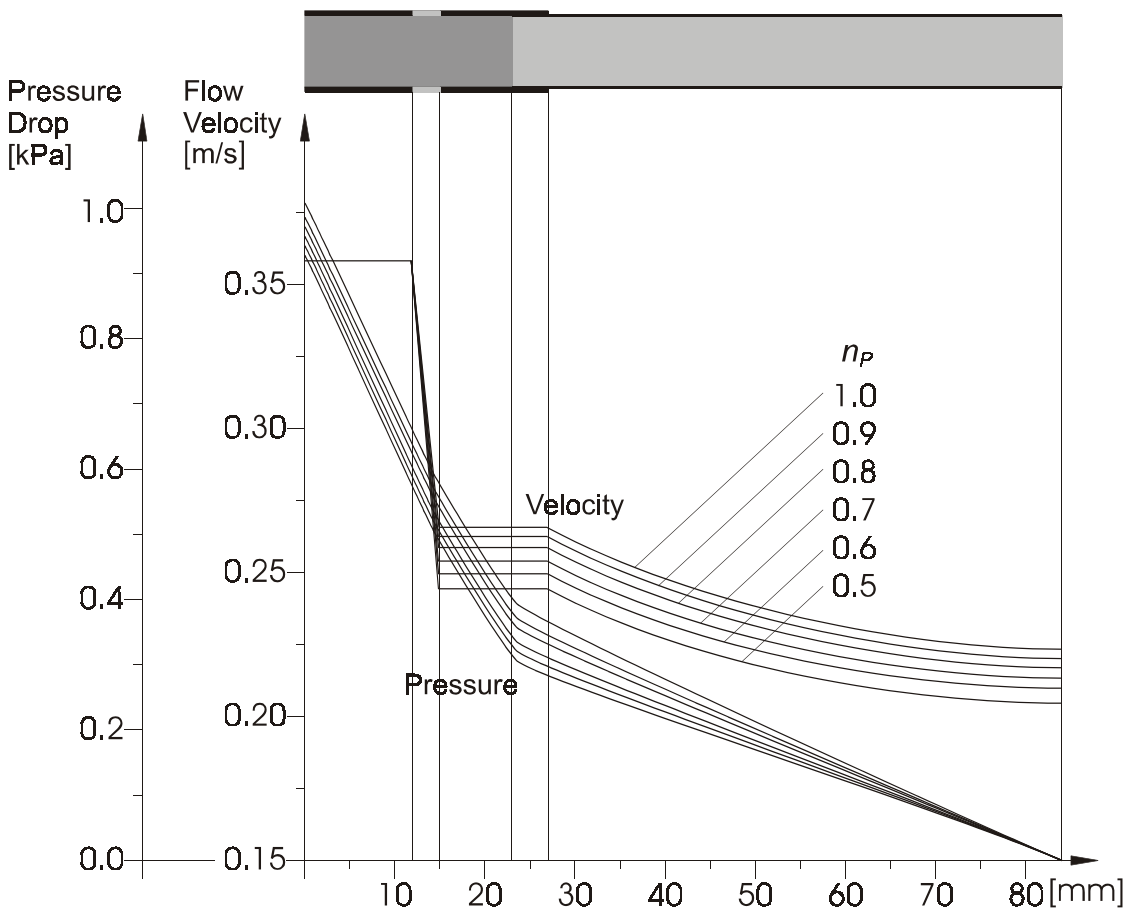

Figure 5.

The influence of nonlinearities in the constitutive equation for the tipping paper on draw resistance and filter ventilation, calculated by a second-order Runge-Kutta method

Nonlinear Constitutive Relation Cigarette Paper

\begin{tabular}{|c|c|}
\hline$n_{P}$ & $\begin{array}{c}\text { Degree of } \\
\text { Filter Ventilation }\end{array}$ \\
\hline 1.0 & $25.7 \%$ \\
0.9 & $25.6 \%$ \\
0.8 & $25.4 \%$ \\
0.7 & $25.2 \%$ \\
0.6 & $24.9 \%$ \\
0.5 & $24.6 \%$ \\
& \\
& Draw \\
$n_{P}$ & Resistance \\
\hline 1.0 & $102.5 \mathrm{~mm}$ WG \\
0.9 & $102.1 \mathrm{~mm}$ WG \\
0.8 & $101.6 \mathrm{~mm}$ WG \\
0.7 & $101.1 \mathrm{~mm}$ WG \\
0.6 & $100.4 \mathrm{~mm}$ WG \\
0.5 & $99.7 \mathrm{~mm}$ WG \\
\hline
\end{tabular}

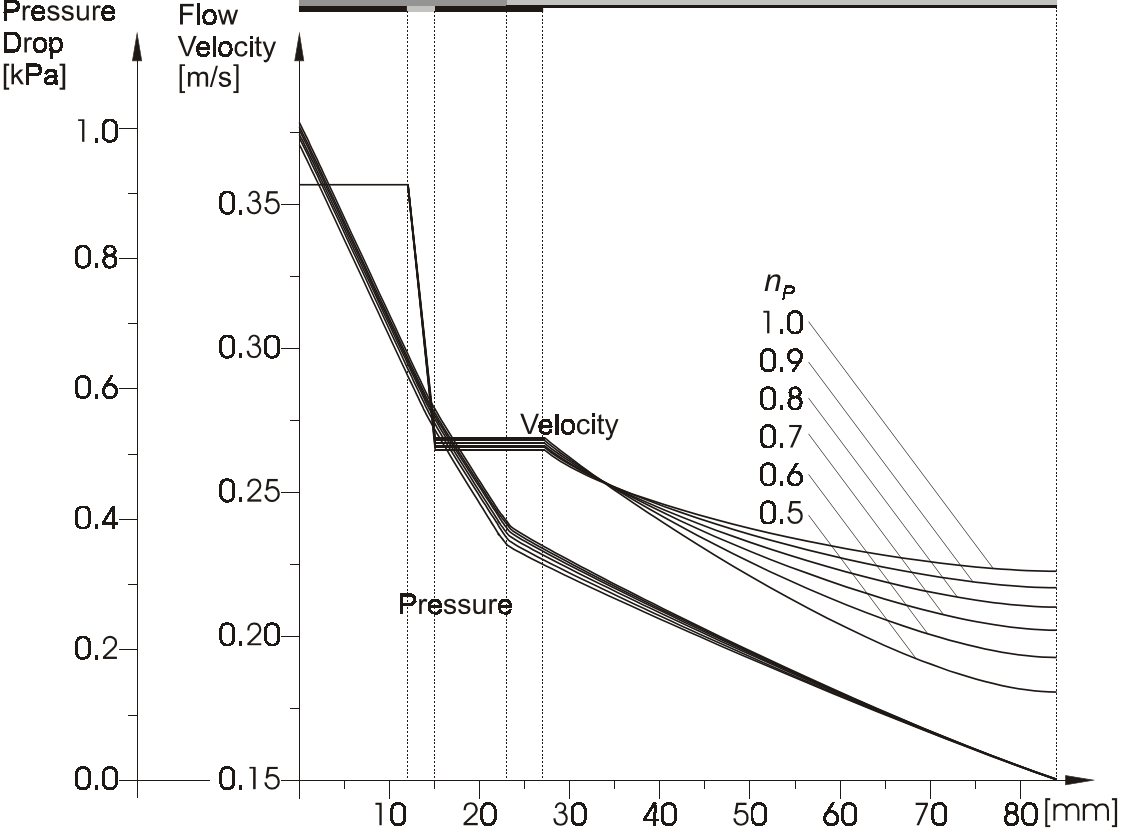

Figure 6.

The influence of nonlinearities in the constitutive equation for the cigarette paper on draw resistance and filter ventilation calculated by a second-order Runge-Kutta method

perforation zone through the paper is higher by a factor of $10 / L_{p}$, with $L_{p}$ in [mm]. Figure 5 shows the velocity and pressure profiles along the cigarette with a nonlinear law for the tipping paper, and a table contains calculated values for the degree of filter ventilation and the draw resistance. For all other components of the cigarette, a linear law has been assumed. Figure 6 shows the same data with a nonlinear pressure drop-flow relation for the ciga- 
Nonlinear Constitutive Relation

Filter

\begin{tabular}{|c|c|}
\hline$m$ & $\begin{array}{l}\text { Degree of } \\
\text { Filter Ventilation }\end{array}$ \\
\hline 1.0 & $25.7 \%$ \\
1.2 & $25.4 \%$ \\
1.4 & $25.1 \%$ \\
1.6 & $24.8 \%$ \\
1.8 & $24.5 \%$ \\
2.0 & $24.2 \%$ \\
\hline & Draw \\
$m$ & Resistance \\
\hline 1.0 & $102.5 \mathrm{~mm}$ WG \\
1.2 & $101.6 \mathrm{~mm}$ WG \\
1.4 & $100.8 \mathrm{~mm}$ WG \\
1.6 & $99.9 \mathrm{~mm}$ WG \\
1.8 & $99.2 \mathrm{~mm}$ WG \\
2.0 & $98.5 \mathrm{~mm}$ WG \\
\hline
\end{tabular}

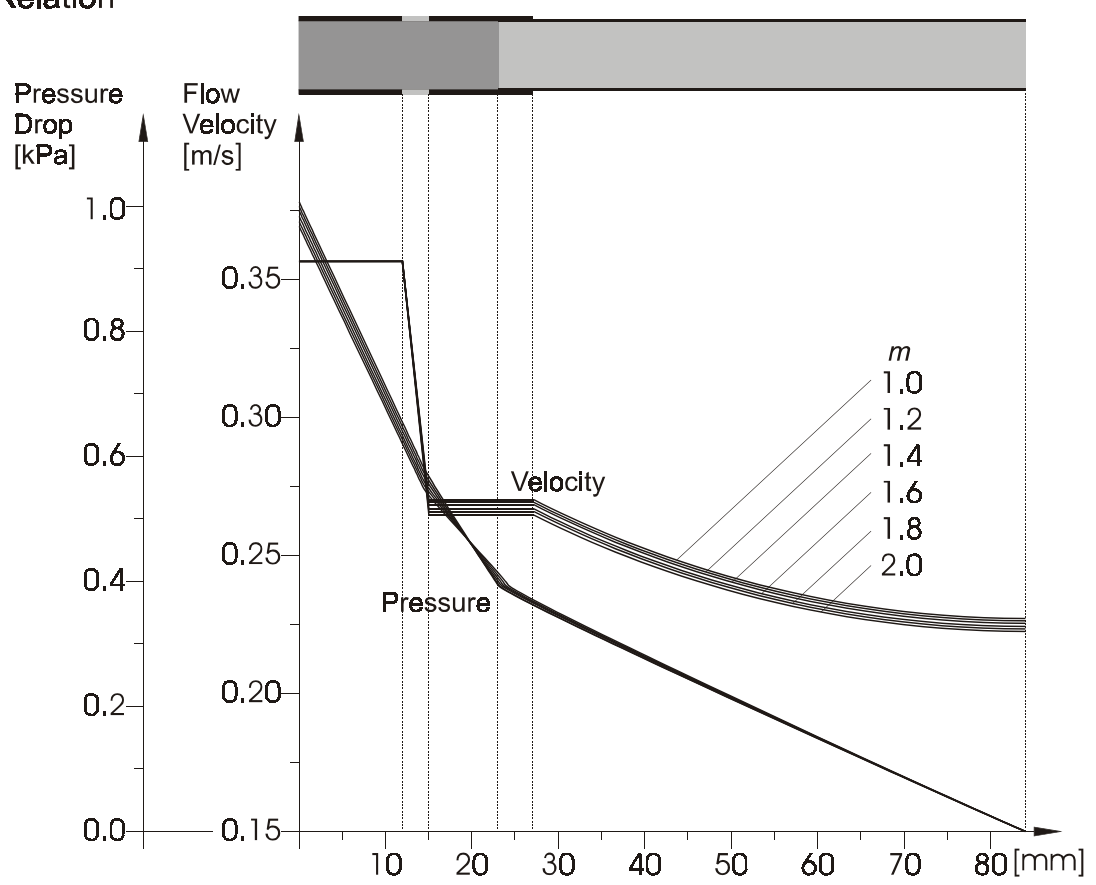

Figure 7.

The influence of nonlinearities in the constitutive equation for the filter on draw resistance and filter ventilation calculated by a second-order Runge-Kutta method

Nonlinear Constitutive
Tobacco Rod
\begin{tabular}{|c|c|}
\hline$m$ & $\begin{array}{l}\text { Degree of } \\
\text { Filter Ventilation }\end{array}$ \\
\hline 1.0 & $25.7 \%$ \\
1.2 & $24.9 \%$ \\
1.4 & $24.2 \%$ \\
1.6 & $23.5 \%$ \\
1.8 & $22.9 \%$ \\
2.0 & $22.4 \%$ \\
\hline & Draw \\
$m$ & Resistance \\
\hline 1.0 & $102.5 \mathrm{~mm}$ WG \\
1.2 & $100.5 \mathrm{~mm}$ WG \\
1.4 & $98.8 \mathrm{~mm}$ WG \\
1.6 & $97.2 \mathrm{~mm}$ WG \\
1.8 & $95.8 \mathrm{~mm}$ WG \\
2.0 & $94.5 \mathrm{~mm}$ WG \\
\hline
\end{tabular}

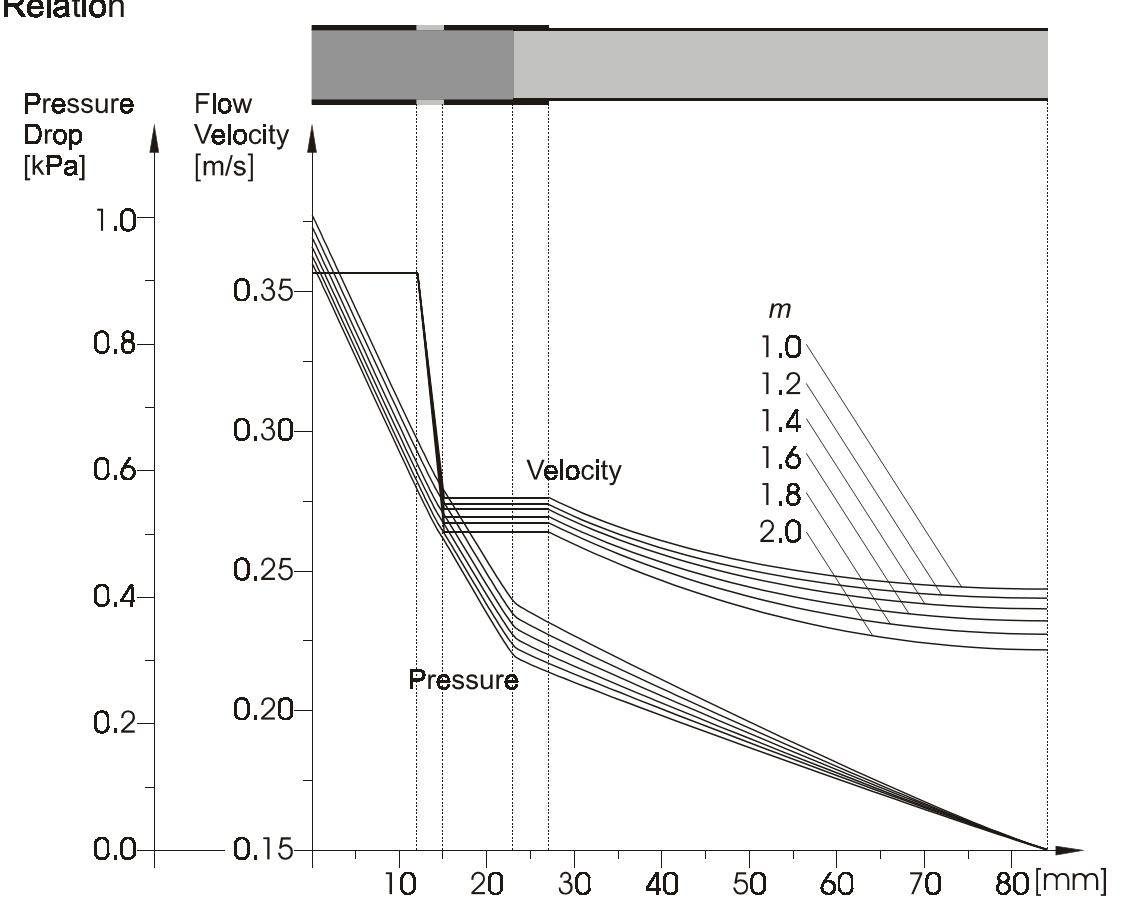

Figure 8.

The influence of nonlinearities in the constitutive equation for the tobacco rod on draw resistance and filter ventilation calculated by a second-order Runge-Kutta method

rette paper instead of the tipping paper. Figure 4 displays several nonlinear constitutive equations for the filter and the tobacco $\operatorname{rod}$ with $m \in$ [1..2]. Again, the tobacco $\operatorname{rod}$ and the filter have a measured pressure drop of $60 \mathrm{~mm}$ $\mathrm{H}_{2} \mathrm{O}$ and $70 \mathrm{~mm} \mathrm{H}_{2} \mathrm{O}$, respectively, and the only way they differ is their behaviour at volumetric flows other than $\pm 17.5 \mathrm{~cm}^{3} / \mathrm{s}$ and $0 \mathrm{~cm}^{3} / \mathrm{s}$. Figures 7 and 8 show the results for the six nonlinear pressure drop-flow relations for the filter and the tobacco rod, respectively. Again, only one component is assumed to be nonlinear. 


\section{DISCUSSION}

As can be seen in Figure 5, the influence of nonlinearities in the tipping paper can be quite significant. The degree of filter ventilation varies from $25.7 \%$ up to $31.8 \%$. The reason for this variation can be seen in Figure 3. While the flow rate through the paper is measured at $1 \mathrm{kPa}$, the actual pressure under the perforation zone is between 0.5 and $0.6 \mathrm{kPa}$. This allows for a variation in flow velocity due to the nonlinearity between approximately 0.055 $\mathrm{m} / \mathrm{s}$ and $0.075 \mathrm{~m} / \mathrm{s}$. This increase of up to $35 \%$ causes a subsequent increase in the degree of filter ventilation. Even in the range of $n_{P}=0.5 . .0 .7$, which is typical for most perforated tipping papers, the degree of filter ventilation varies between $29.0 \%$ and $31.8 \%$, about $10 \%$ of its nominal value. The effect on draw resistance is far less significant. The nonlinearity causes a drop from $102.5 \mathrm{~mm} \mathrm{H}_{2} \mathrm{O}$ down to $97.6 \mathrm{~mm} \mathrm{H}_{2} \mathrm{O}$, which is a reduction of about $5 \%$.

In Figure 6 it is shown that nonlinearities in the constitutive equation of the cigarette paper have only a minor influence on the degree of filter ventilation and also on draw resistance. Yet the degree of rod ventilation, defined as the ratio of the volumetric flow entering the cigarette through the cigarette paper to the total volumetric flow, i.e., $V_{\text {Rod }}=(v(z=27)-v(z=84)) / v_{\text {tot }}$, varies by a factor of 2 between approximately $10 \%$ and $20 \%$. This variation is the more important because it cannot be detected by measurements of filter ventilation and draw resistance alone, but it does have an influence on smoke yields.

For nonlinear filter materials, Figure 7, the situation is much better. Only small reductions in the degree of filter ventilation and draw resistance can be observed and additionally, real filter material is very homogeneous and is almost linear in its behaviour so that this influence can usually be neglected.

For the tobacco rod, the situation is again more interesting. Variations in the exponent $m$ between 1.0 and 2.0 cause variations in the degree of filter ventilation between $25.7 \%$ and $22.4 \%$, a lot smaller than variations due to the tipping paper, but still more than $10 \%$. The draw resistance will drop from $102.5 \mathrm{~mm} \mathrm{H}_{2} \mathrm{O}$ to $94.5 \mathrm{~mm}$ $\mathrm{H}_{2} \mathrm{O}$ as $m$ increases from 1.0 to 2.0 .

It must be noted, however, that it is not correct to conclude from these calculations that macro-laser perforated tipping paper $n_{P}=0.5$ should, in terms of filter ventilation, be more efficient than an electro-perforated tipping paper $n_{P}=0.7$ of the same permeability at $1 \mathrm{kPa}$. Macro-laser perforations are usually concentrated in a few narrow tracks of perforation with an extremely high "local" permeability at the perforation track and almost no permeability in between, while electro-perforated tipping paper shows an evenly distributed permeability over the whole perforation width. Thus, the plug wrap paper, seen as a resistance in series with the tipping paper, will cause a greater reduction of overall permeability for macro-laser perforated tipping because a smaller area of plug wrap paper is available for the air flow.
Depending on the specific perforation, this effect might even dominate over the nonlinearity.

\section{CONCLUSIONS}

A new nonlinear constitutive equation for the flow in porous media and a model for the cigarette using this law has been derived and applied to the stationary flow in unlit cigarettes to investigate its influence on the degree of filter ventilation and draw resistance. By numerical calculations it was shown that this influence is significant, especially for the tipping paper, where nonlinearities caused a variation in the degree of filter ventilation of up to $20 \%$ of the nominal value. Thus, it does not suffice to know the permeability of the paper only at a pressure difference of $1 \mathrm{kPa}$, as most cigarettes will have a smaller pressure difference at the perforation zone. If one wants to predict the pressure drop and the degree of filter ventilation for a new cigarette with higher than standard precision or search for sources of variations in these quantities, the nonlinearity of the constitutive equations is a point worthy of consideration.

\section{REFERENCES}

1. Synge, J.L. and A. Schild: Tensor calculus; Dover Publications, Inc., New York, 1978.

2. Bear, J.: Dynamics of fluids in porous media; Dover Publications, Inc., New York, 1972.

3. Spurk, J.H.: Fluid mechanics; Springer-Verlag, Berlin, 1997.

4. Meyer-Abich, K.M.: Die Strömungsverhältnisse in Cigaretten; Beitr. Tabakforsch. 3 (1966) 307-329.

5. Selke, W.A. and J.H. Mathews: The permeability of cigarette papers and cigarette ventilation; Beitr. Tabakforsch. Int. 9 (1978) 193-200.

6. Schneider, W.A. Schlüter, and F. Seehofer: The effects of the properties of materials in a cigarette on filter ventilation; Beitr. Tabakforsch. Int. 12 (1984) 123-136.

7. Baker, R.R.: The viscous and inertial flow of air through perforated papers; Beitr. Tabakforsch. Int. 14 (1989) 253-260.

8. Gaißer, H.: Die stationäre Strömung in der Cigarette und deren Einfluß auf den Zugwiderstand; Beitr. Tabakforsch. Int. 15 (1992) 93-122.

9. Gaißer, H.: Fluiddynamik in der Cigarette und deren Einfluß auf den Zugwiderstand; Beitr. Tabakforsch. Int. 16 (1994) 11-46.

10. Dwyer, R.W. and P. Chen: Prediction of pressure drop and ventilation in a lit cigarette; Beitr. Tabakforsch. Int. 18 (1999) 205-211.

11. Riley, D.S., D.G. Drake, R.R Baker, and D.P. Robinson: Non-Darcy flow and diffusion in a tobacco rod; Physico Chemical Hydrodynamics 7 (1986) 255-279. 
12. Baker, R.R and D.P. Robinson: Non-Darcy flow and diffusion in a tobacco rod; Proceedings of the International Conference on the Physical and Chemical Processes occurring in a Burning Cigarette, WinstonSalem, NC, USA, 1987, pp. 355-408.

13. Keith, C.H.: Pressure drop-flow relationships in cigarette filter rods and tobacco columns Beitr. Tabakforsch. Int. 11 (1982) 115-121.

14. Palmade, P.: Contribution to the study of flows in cigarettes; Ann. du Tabac 17 (1979) 37-48.
15. Holzapfel, G.A.: Nonlinear solid mechanics; John Wiley \& Sons, New York, 2000, 192-196.

Address for correspondence:

Bernhard Eitzinger

F\&E / Tann Papier GmbH

Fabrikstraße 20

A-4050 Traun

Austria 